\title{
Asymmetric-detection time-stretch optical microscopy (ATOM) for high-contrast and high-speed microfluidic cellular imaging
}

\author{
Terence T. W. Wong ${ }^{\mathrm{a}}$, Andy K. S. Lau ${ }^{\mathrm{a}}$, Matthew Y. H. Tang ${ }^{\mathrm{b}}$, Kenneth K. Y. Ho ${ }^{\mathrm{b}}$, \\ Kenneth K. Y. Wong ${ }^{\mathrm{a}}$, Anderson H. C. Shum ${ }^{\mathrm{b}}$, and Kevin K. Tsia ${ }^{\mathrm{a}, *}$ \\ ${ }^{a}$ Department of Electrical and Electronic Engineering, The University of Hong Kong, Pokfulam \\ Road, Hong Kong, China; ${ }^{b}$ Department of Mechanical Engineering, The University of Hong Kong, \\ Pokfulam Road, Hong Kong, China; ${ }^{*}$ Corresponding author e-mail address: tsia@ hku.hk; phone 852 \\ 2857-8486; fax 852 2559-8783; www.eee.hku.hk/ alphahku
}

\begin{abstract}
High-throughput cellular imaging is acclaimed as captivating yet challenging in biomedical diagnostics. We have demonstrated a new imaging modality, asymmetric-detection time-stretch optical microscopy (ATOM), by incorporating a simple detection scheme which is a further advancement in time-stretch microscopy - a viable solution to achieve high-speed and high-throughput cellular imaging. Through the asymmetric-detection scheme in ATOM, the time-stretch image contrast is enhanced through accessing to the phase-gradient information. With the operation in the $1 \mu \mathrm{m}$ wavelength range, we demonstrate high-resolution and high-contrast cellular imaging in ultrafast microfluidic flow (up to $10 \mathrm{~m} / \mathrm{s}$ ) by ATOM - achieving an imaging throughput equivalent to $\sim 100,000 \mathrm{cells} / \mathrm{sec}$.
\end{abstract}

Keywords: time-stretch imaging, high-speed microfluidic, cell imaging, ultrafast frame rate, biophotonics, imaging flow cytometry, high-throughput

\section{INTRODUCTION}

Having the ability of accessing to the morphological information of the cells and thus facilitating better cellular identification/classification with high statistical accuracy, optical microscopy is of great value when it is incorporated in high-throughput cell screening - a routine utilized in clinical diagnostics and basic life science research. Specifically, high-speed optical imaging techniques are essential in order to maintain the required high-throughput. Yet, current approaches have largely been restricted by a trade-off between throughput and image quality (i.e. screening accuracy). Very often, scaling the imaging speed in optical microscopy is achieved at the expense of image quality, primarily dictated by image contrast, image resolution, and detection sensitivity - a common predicament for advancing high-speed and high-throughput cellular imaging. It is exemplified by that the current state-of-the-art imaging flow cytometers have to scale down the imaging throughput to $\sim 1000$ cells/sec, compared with the throughput of $\sim 100,000$ cells/sec of the classical non-imaging flow cytometers [1].

\section{Asymmetric-detection time-stretch optical microscopy (ATOM)}

To this end, time-stretch microscopy, a new optical imaging modality overcomes the intrinsic tradeoff between sensitivity and imaging speed in the commercial imaging flow cytometer, has recently been developed for ultrafast imaging. With the conversion of the image spatial coordinates encoded in the spectrum of a broadband and ultrashort optical pulse (fs - ps) into a serial temporal data series, time-stretch microscopy has been shown to perform high-throughput image-based cell screening, which could be an ideal complementary tool to typical multiparametric flow cytometry, primarily enjoying the ultrafast image retrieval (> $\mathrm{MHz}$ frame rate). However, current time-stretch microscopy has so far mostly been operated in bright-field (BF) imaging mode in longer wavelength range (1500 - 1600

Imaging, Manipulation, and Analysis of Biomolecules, Cells, and Tissues XII, edited by

Daniel L. Farkas, Dan V. Nicolau, Robert C. Leif, Proc. of SPIE Vol. 8947, 89471D

(C) 2014 SPIE $\cdot$ CCC code: 1605-7422/14/\$18 - doi: 10.1117/12.2038952 
nm) [2-4], which hinders accurate image-based cell recognition and screening and is thus hard to reveal high-contrast and high-resolution morphology of the stain-free and transparent cells. As a result, effective use of time-stretch imaging to-date only has been limited to microparticle or cell screening in high-speed flow with trivial size and shape differences, especially when the targeted cells are labeled with contrast agents [3]. In this regard, we present a new imaging approach called asymmetric-detection time-stretch optical microscopy (ATOM), as a solution to capture label-free but high-contrast image of the transparent cells in a PDMS microfluidic flow imaging at ultrahigh imaging flow speed of 10 $\mathrm{m} / \mathrm{s}$. The flow speed is equivalent to an imaging throughput of $\sim 100,000$ cells $/ \mathrm{sec}$ - order-of-magnitude higher than the state of the art imaging flow cytometers $(\sim 1,000$ cells/sec $)$. Together with the operation at a shorter $1 \mu \mathrm{m}$ wavelength regime, ATOM thus represents a significant advancement in bringing the essential imaging metrics - high resolution and high contrast - to high-speed time-stretch imaging, making it a genuinely appealing platform for realizing high-throughput image-based cellular assay.

\section{Working principles}

The core idea of ATOM is to generate the enhanced-contrast time-stretch images by asymmetrically detecting the spectrally-encoded pulses prior to the time-stretch process. The single-angle ATOM image can be achieved by coupling off-axis light into the optical fiber core at the fiber collimator, which also acts as a confocal pinhole of the ATOM system - resembling the contrast-enhancement mechanism applied in Schlieren or phase-gradient contrast imaging [5-9]. More importantly, we can split and time-multiplex the spectrally-encoded pulses into the multiple replica. Different off-axis coupling angle yields different phase-gradient contrast. In this paper, we have demonstrated that by time-multiplexing two single-angle ATOM images with opposite phase-gradient contrasts, two distinct and decoupled contrasts from the same unstained cell can be achieved: one with differential (enhanced) phase-gradient contrast and another with absorption contrast, simultaneously.

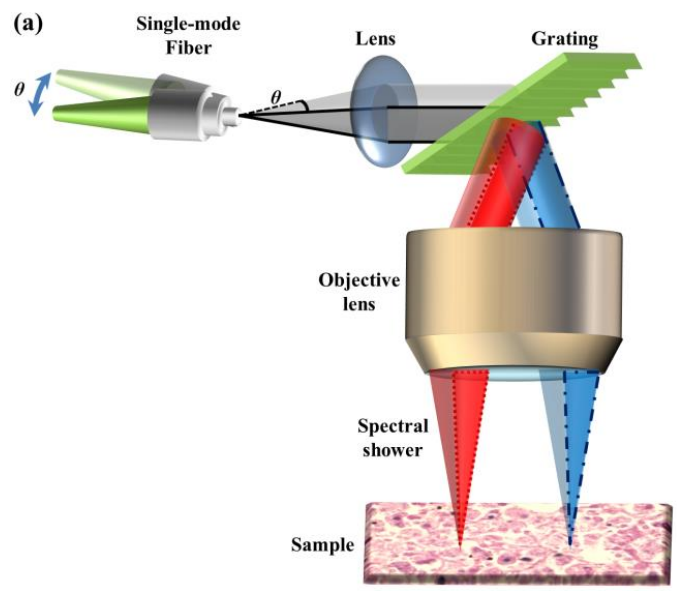

(b)
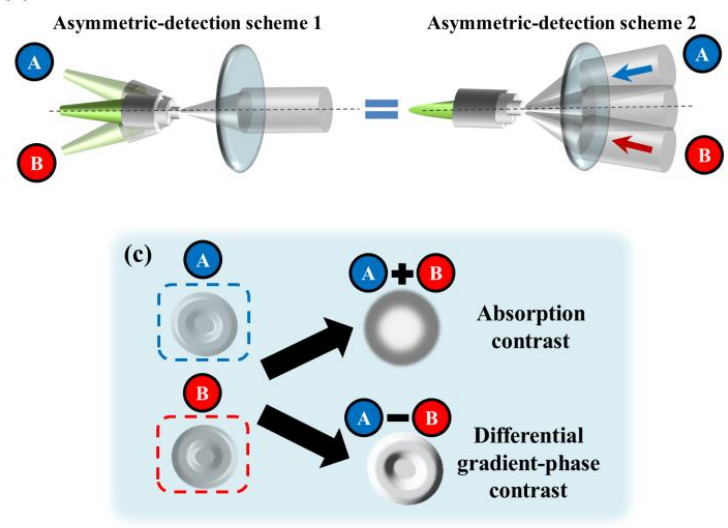

Figure 1 Working principle of ATOM for enabling phase-gradient contrast. (a) In typical BF imaging mode of time-stretch optical microscopy, the spatial information of the specimen is first encoded to the spectral shower of a broadband pulsed laser by a diffraction grating. The spectrally-encoded pulsed beam is then coupled on-axis into the fiber core of the dispersive fiber for the afterward time-stretch process. In ATOM, the spectrally-encoded pulsed beam is coupled off-axis into the fiber core to introduce an oblique detection angle $\theta$ between the fiber axis and the beam propagation axis, as the numerical aperture (NA) of fiber core limits the cone of the coupling light asymmetric (darker gray area of the coupling beam). In effect, it is equivalent to partially blocking the beam detection path, and thus to asymmetrically capture the light from the sample - giving rise the three-dimensional (3D) appearance, which is proportional to the phase-gradient. Along the whole spectrum, same degree of phase-gradient contrast for all wavelengths is preserved (the enclosed areas of both the red and blue components), this can be understood by considering that all the encoded wavelengths (e.g. blue and red components depicted in the figure) are recombined by the same diffraction grating and are collected by the fiber within the same asymmetric coupling light cone. (b) Asymmetric-detection scheme 1 and 2 show the same result even though the component axis to be tilted is different, i.e. the fiber core axis and the beam propagation axis respectively, this is solely caused by again the limited NA of fiber core, which results in the same acceptance cone of coupled light if the oblique angle is the same. (c) In coupled beams A and B in (b), the single-angle ATOM images show opposite phase-gradient contrast, which can be further utilized to compute the dual-angle (difference) ATOM image and dual-angle (sum) ATOM image showing the differential gradient-phase contrast and absorption contrast of the same cell respectively. 


\section{Experimental Setup}

Based on our home-built ytterbium-doped mode-locked laser (repetition rate: $26 \mathrm{MHz}$; center wavelength: $1064 \mathrm{~nm}$; bandwidth: $\sim 10 \mathrm{~nm}$ ), our current ATOM system operates in the $1-\mu \mathrm{m}$ wavelength regime to achieve a better diffraction-limited resolution $(\sim 1.2 \mu \mathrm{m})$. With the use of a diffraction grating (groove density: 1200 lines $/ \mathrm{mm}$ ), a one-dimensional spectral shower is then generated and focused onto the sample plane through an objective lens (NA: 0.66). A double-pass illumination scheme is employed optimizing for unstained and transparent cells imaging by introducing another identical objective lens (NA: 0.66) behind the sample. The reflected image-encoded pulse is then split into two arms (A and B in Fig. 2 (a)) to introduce different time-delays and oblique detection angle at the fiber collimator. Through a dispersive fiber module, which consists of a $5 \mathrm{~km} \mathrm{SMF} \mathrm{(Nufern} \mathrm{1060XP)} \mathrm{and} \mathrm{a} 5 \mathrm{~km} \mathrm{SMF-28} \mathrm{(as}$ a few-mode fiber [10], the reflected broadband laser pulse is time-stretched with a group velocity dispersion of $\sim 0.35$ $\mathrm{ns} / \mathrm{nm}$. Implementing an in-line optical amplification using a fiber-based semiconductor optical amplifier (SOA), the dispersed temporal serial data-stream is amplified with an optical gain of $\sim 10 \mathrm{~dB}$ for compensation of fiber loss. Finally, the signal is detected by a single-pixel photo-detector $(8 \mathrm{GHz})$ and a real-time oscilloscope $(40 \mathrm{GS} / \mathrm{s})$. Due to the time-delay introduced before coupling is shorter than a period of the laser repetition, the two asymmetrically detected signals are time-multiplexed without sacrificing the frame rate. This highly enables ATOM favorable for ultra-high speed flow cell imaging.

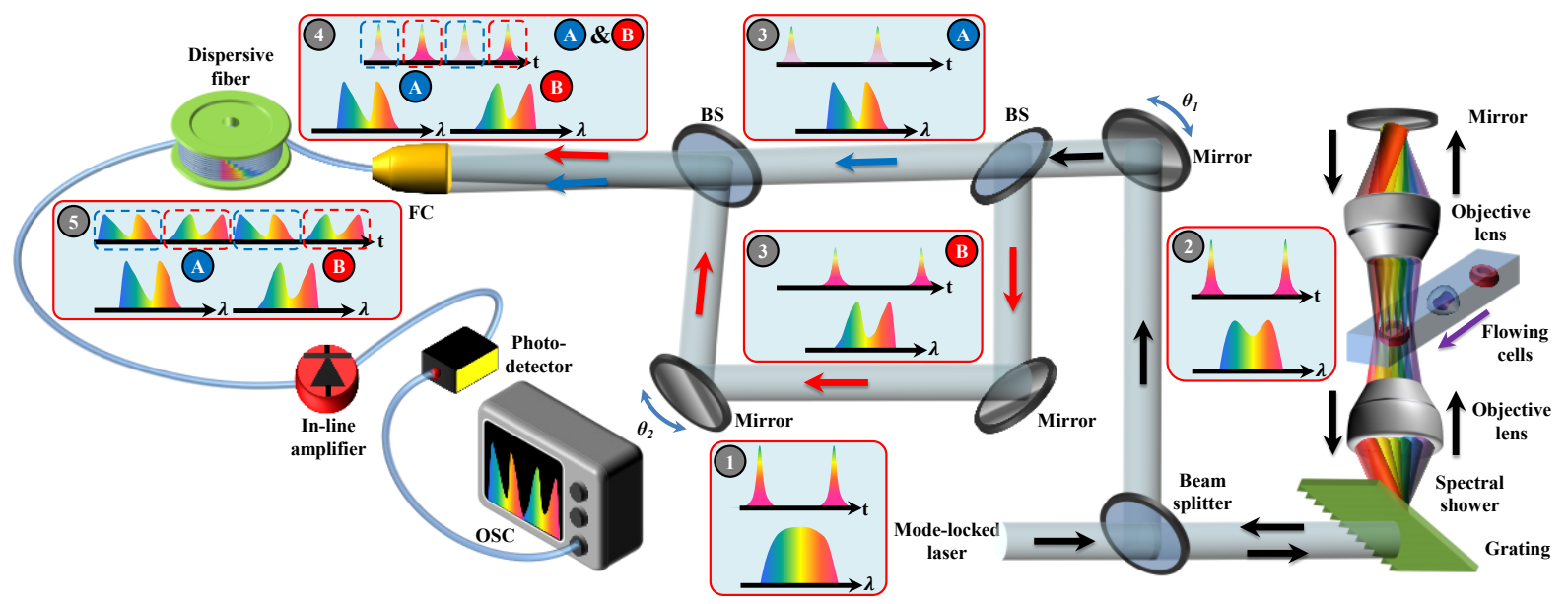

Fig. 2: General schematic of an ATOM system. (a) A diffraction grating is used to spatially dispersed a broadband light pulsed beam (the temporal pulse train and the corresponding spectrum are shown in box 1) to generate a 1D spectral shower. After being focused by an objective lens for illumination, different wavelengths are focused on different locations on the flowing cells. Having the double-pass transmission configuration, the reflected spectrally-encoded pulses, which map the spatial information of the sample into the spectrum, are then recombined to a collimated pulsed beam by the same grating (see box 2). The fiber collimator couples the two split and then recombined beams (A and B) to introduce equal but opposite angles in these two beams, each of which generates a single-angle ATOM beam revealing opposite phase-gradient contrast as illustrated in scheme 2 in Fig. 1(b) (see boxes 3). Beam B here is time-delayed so that they are multiplexed in time without temporal overlap (see box 4). The time-multiplexed signals then undergo the time-stretch process to perform wavelength-to-time mapping and an in-line optical image amplification is implemented to compensate the intrinsic loss in the fiber (see box 5). A single-pixel photodetector and a real-time oscilloscope are used for signal acquisition. It is worth noting that each single-shot line-scan of the time-stretch signal corresponds to the pulse period of the mode-locked laser. An ATOM image is reconstructed by digitally stacking the 1D line-scans.

\section{RESULTS}

\section{Basic performance of ATOM}

We first demonstrate the central concept of ATOM by imaging the unstained normal hepatocyte cell (MIHA), fixed on a glass slide and scanned orthogonal to the spectral shower direction on a motorized translational stage, with two opposite asymmetric-detection angles, i.e. two opposite phase-gradient contrasts (Figs. 3(a)-(b)). The two-dimensional (2D) images are obtained by digitally stacking the 1D line-scans. The effective single-shot line-scan rate is $1 \mathrm{MHz}$ by taking 
averaging of 25 sequential line-scans. With the tunability on the asymmetric-detection angles, it is worth to mention the shadow cast of the images can be switched to the opposite side of the MIHA cell. By simply computing the sum and difference of the two time-multiplexed opposite-contrast ATOM images, both the absorption and the differential (enhanced) phase-gradient-contrast images of the MIHA cell can be obtained at ultrahigh speed (line scan time $\sim 4 \mathrm{~ns}$ ) without the downgrade of line-scan rate, respectively (Figs. 2(c) and (d)). To compare the performance of ATOM with the BF imaging mode of time-stretch microscopy under high-speed flow $(8 \mathrm{~m} / \mathrm{s})$, Fig. 3(e) shows the contrast enhancement of corresponding flowing MIHA cell image in a PDMS microfluidic channel, through microfluidic inertial flow. The 2D images are here obtained by continuous recording the real-time 1D line-scan (line-scan rate: $26 \mathrm{MHz}$ (governed by the repetition rate of the laser)), which is naturally provided by the unidirectional cell flow.

(a) Single-angle ATOM (A)

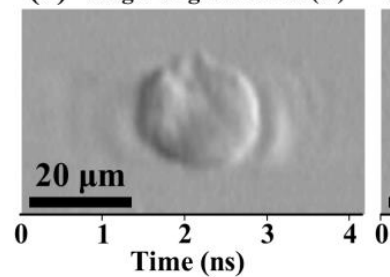

(c) Dual-angle ATOM (A+B)

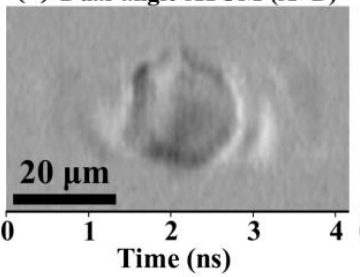

(b) Single-angle АТОм (B)

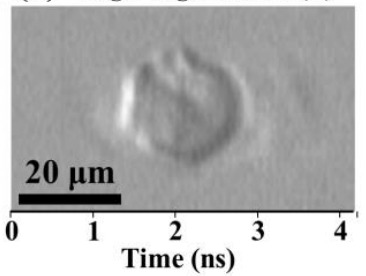

(d) Dual-angle ATOM (A-B)

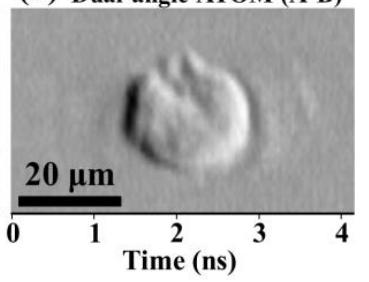

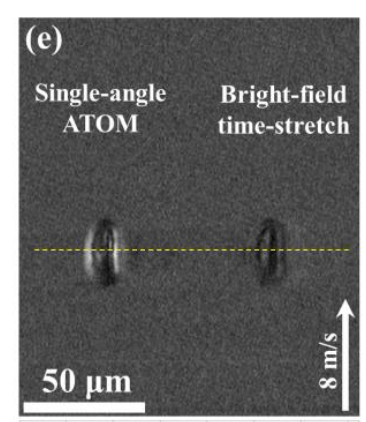

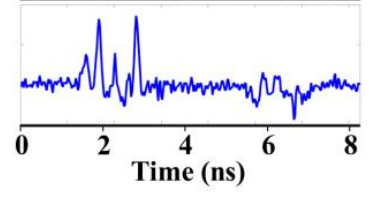

Fig. 3: (a,b) Two opposite single-angle ATOM images of a normal hepatocyte (MIHA), which show the opposite phase-gradient contrasts, respectively. (c) Dual-angle ATOM image showing absorption-contrast of the MIHA, by computing the sum of the two images (i.e. A+B). (d) Dual-angle ATOM image showing differential phase-gradient-contrast of the MIHA, by computing the difference of the two images (i.e. A-B). Each image is reconstructed by 70 line-scans translating in a perpendicular direction to the spectral shower direction, each line scan of the image is captured within $\sim 4$ ns (i.e. duty cycle is $\sim 10 \%$ ). (e) Demonstration of the contrast enhancement in ATOM by comparing the single-angle ATOM with the bright-field time-stretch imaging scheme in ultrahigh-speed flow imaging $(\sim 8 \mathrm{~m} / \mathrm{s})$ of the MIHA in a PDMS-based microfluidic channel. Note that both images of the same cell are obtained simultaneously by the time-multiplex scheme. Bottom inset shows the line profile along the yellow dashed line.

\section{Imaging of blood cells}

We also captured the ATOM images of the acute leukemic monocytic (THP-1) cells (Figs. 4(b) - (d)) and normal human blood cells, from fresh blood obtained from a healthy donor (Figs. 4(f) - (h)), flowing in the same microfluidic channel at a speed as high as $10 \mathrm{~m} / \mathrm{s}$ - equivalent to an imaging throughput of $\sim 100,000$ cells $/ \mathrm{sec}$, a comparable throughput of conventional non-imaging flow cytometers. From the differential phase-gradient contrast images of the THP-1 cells in ATOM we can visualize the nuclei (indicated by arrows in Figs. 4(c)) - imaging such sub-cellular structure without extra contrast-enhancing label is yet to be demonstrated in ordinary BF time-stretch imaging. The corresponding differential interference contrast (DIC) image of THP-1 cells is shown in Fig. 4(a), indicating ATOM could reveal one of the distinctive features in THP-1 cells. For the whole blood sample, ATOM is able to identify the biconcave disk shape of the red blood cells (RBCs) and can differentiate them from the swelled RBCs in the flow, which are in either spherical or elliptical shapes (Fig. 4(g)). The corresponding DIC image of the whole blood on glass slide is shown in Fig. 4(e), showing the normal RBCs structure and swelled RBCs. The results of ATOM presented here are of great significance for advancing time-stretch imaging for high-throughput imaging flow cytometry with high statistical precision (enabled by high-contrast images). 
THP-1

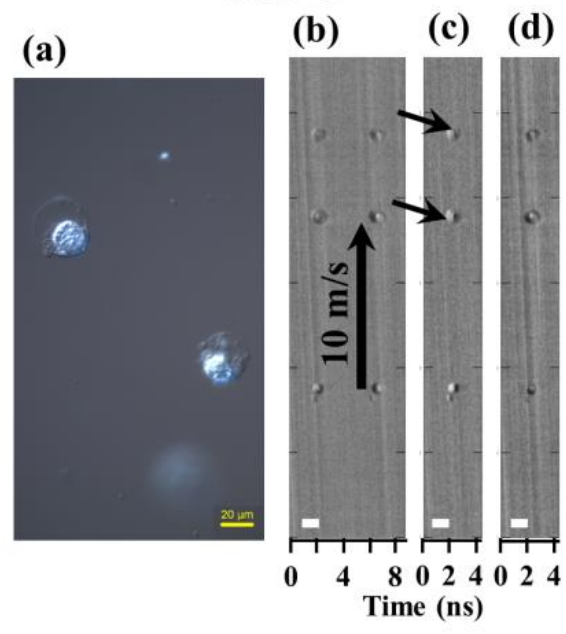

\section{Whole blood}

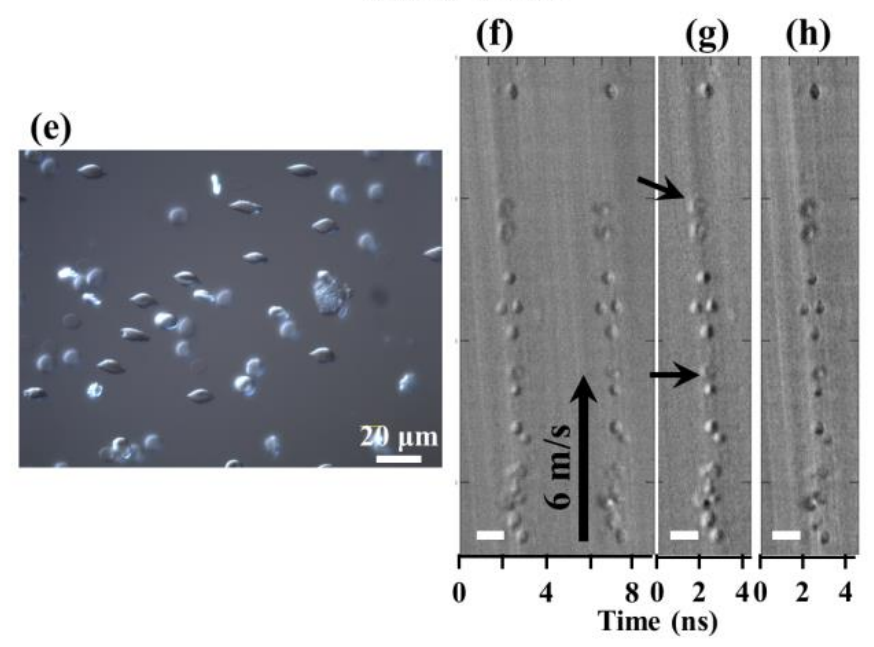

Fig. 4: Imaging acute leukemic monocytic cells (THP-1, a-d) and normal human blood cells (from whole blood, e-h). (a) DIC image of THP-1 cells. (b) Time-multiplexed single-angle ATOM image of THP-1 cells flowing at $10 \mathrm{~m} / \mathrm{s}$. (c) Dual-angle (differential phase-gradient-contrast) ATOM image of THP-1 cells by subtraction of the two time-multiplexed single-angle ATOM images in (b). (d) Dual-angle (absorption-contrast) ATOM image by summation of the two time-multiplexed single-angle ATOM images in (b). (e) DIC image of fresh blood. (f) Time-multiplexed single-angle ATOM image of blood cells flowing at $6 \mathrm{~m} / \mathrm{s}$. (g) Dual-angle (differential) ATOM image in (f). (h) Dual-angle (absorption) ATOM image in (f). Scale bars are all in $20 \mu \mathrm{m}$.

\section{SUMMARY}

We have demonstrated a new ultrafast optical imaging technique called ATOM, which delivers high-contrast cellular imaging in an ultrahigh-speed microfluidic flow up to $10 \mathrm{~m} / \mathrm{s}$ - orders-of-magnitude faster than the typical optofluidic cellular imaging techniques $(\sim \mathrm{mm} / \mathrm{s})$, i.e. the equivalent imaging throughput is 100,000 cells/s. With the ordinary BF imaging mode of time-stretch microscopy, it has been limited to effectively perform high-throughput cell screening applications, which shows only low image contrast - hindering image-based cellular identification and classification. By applying the asymmetric-detection scheme, ATOM can exploit stain-free phase-gradient contrast of the cells by. With the duty cycle of a time-stretch temporal serial waveform of $\sim 10 \%$, ATOM can further access to both the differential (enhanced) gradient-phase contrast and the absorption contrast simultaneously through time-multiplexing two spectrally-encoded pulses - without compromising the final imaging speed (> tens of $\mathrm{MHz}$ ).We also stress that the ATOM system reported here is operated in the shorter near infrared window, $\sim 1 \mu \mathrm{m}$, in contrast to most of the prior work on time-stretch imaging which operates in the telecommunication band. It is not only favorable for imaging with higher diffraction-limited resolution $(\sim 1.2 \mu \mathrm{m})$, but also opens up a wider scope of biophotonic applications, especially for in-vivo imaging.

Combining the unique feature of dual-angle (differential) ATOM, i.e. the enhanced phase-gradient image contrast, together with the higher diffraction-limited resolution, ATOM realizes accurate image-based cell identification with ultrahigh throughput. This is particularly exemplified by its feasibility of imaging the characteristic cellular features of individual stain-free normal human blood cells as well as leukemic cells by ATOM.

The results of ATOM presented here are of great significance for advancing time-stretch imaging for high-throughput imaging flow cytometry with high statistical precision. It is particularly envisaged for rare cell screening in early metastasis detection, or post-chemotherapy detection of the residual cancer cells, a concept called minimal residual disease (MRD) detection [11]. Future development will be focused on real-time ATOM and automated image recognition and analysis, by integrating the system with parallel digital signal processing based on field-programmable gate array (FPGA) or graphic processing unit (GPU). As a result, it would be a powerful tool for high-throughput image-based cellular assay, particularly complementary to the multiparametric analysis of the existing non-imaging flow cytometry. 


\section{ACKNOWLEDGEMENTS}

We thank Hilary K. Y. Mak for preparing the MIHA, BEL-7402 and HeLa cell lines. We also thank Joseph D. F. Robles for preparing the whole blood sample and the acute leukemic monocytic cells (THP-1). This work was partially supported by grant from the Research Grants Council of the Hong Kong Special Administrative Region, China (Project No. HKU 7172/12E, HKU 717510E, HKU 717911E, HKU 720112E) and University Development Fund of HKU.

\section{REFERENCES}

[1] Basiji, D. A., Ortyn, W. E., Liang, L., Venkatachalam, V., \& Morrissey, P. "Cellular Image Analysis and Imaging by Flow Cytometry," Clin Lab Med. 27, 653-670 (2007).

[2] Goda, K., Tsia, K. K. \& Jalali, B. "Serial time-encoded amplified imaging for real-time observation of fast dynamic phenomena," Nature 458, 1145-1149 (2009).

[3] Goda, K. et al. "High-throughput single-microparticle imaging flow analyzer," Proc. Natl Acad. Sci. USA 109, 11630-11635 (2012).

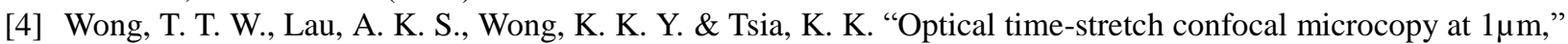
Opt. Lett. 37, 3330-3332 (2012).

[5] Kachar, B. "Asymmetric illumination contrast: a method of image formation for video light microscopy," Science 227, 766-768 (1985).

[6] Lowenthal, S. \& Y. Belvaux, Y., "Observation of phase objects by optically processed Hilbert transform," Appl. Phys. Lett. 11, 49-51 (1967).

[7] Mehta, S. B. \& Sheppard, C. J. R., "Quantitative phase-gradient imaging at high resolution with asymmetric illumination-based differential phase contrast," Opt. Lett. 34, 1924-1926 (2009).

[8] Ford, T. N., Chu, K. K. \& Mertz J., "Phase-gradient microscopy in thick tissue with oblique back-illumination," Nat. Methods, 9, 1195-1197 (2012).

[9] Parthasarathy, A. B., Chu, K. K., Ford, T. N. \& Mertz, J., "Quantitative phase imaging using a partitioned detection aperture," Opt. Lett. 37, 4062-4064 (2012).

[10] Qiu, Y., Xu, J., Wong, K. K. Y., and Tsia, K. K., "Exploiting few mode-fibers for optical time-stretch confocal microscopy in the short near-infrared window," Opt. Express 20, 24115-24123 (2012).

[11]Bene, M. C. \& Kaeda, J. S., "How and why minimal residual disease studies are necessary in leukemia: a review from $\mathrm{WP}_{10}$ and $\mathrm{WP}_{12}$ of the European LeukaemiaNet," haematologica 94, 1135-1150 (2009). 